\title{
BalloonAscent: 3-D Simulation Tool for the Ascent and Float of High-Altitude Balloons
}

\author{
Rodger E. Farley* \\ NASA / Goddard Space Flight Center, Greenbelt, Maryland, 20771
}

\begin{abstract}
BalloonAscent is a new user-friendly software tool, which simulates the vertical and horizontal motions of high-altitude balloons such as are flown by the National Scientific Balloon Facility. The formulations are generalized as to allow simulated balloon flight in other atmospheres on different planets. This paper will describe the theoretical basis and how the tool is used to predict ascent behavior and float behavior under varying environmental conditions.
\end{abstract}

\section{Nomenclature}

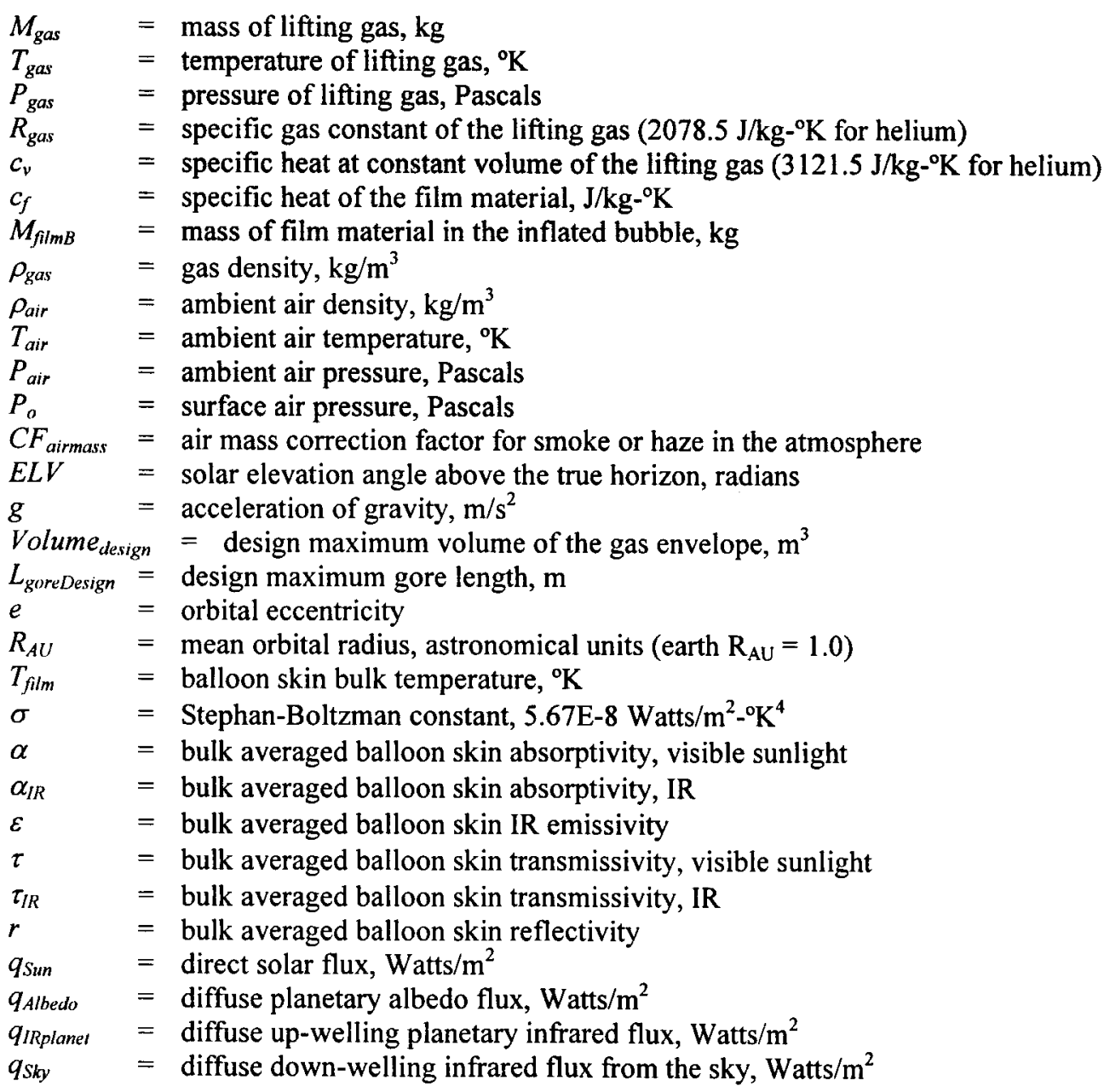

\footnotetext{
-Aerospace Engineer, Mechanical Systems Center, Code 543, NASA / GSFC
} 


\section{Introduction}

$\mathrm{T}$ HE BalloonAscent balloon flight simulation code represents a from-scratch development using Visual Basic 5 as the software platform. The simulation code is a transient analysis of balloon flight, predicting the skin and gas temperatures along with the 3-D position and velocity in a time and spatially varying environment. There are manual and automated controls for gas valving and the dropping of ballast. Also, there are many handy calculators, such as appropriate free lift, and steady-state thermal solutions with temperature gradients.

The strength of this simulation model over others in the past is that the infrared environment is deterministic rather than guessed at. The ground temperature is specified along with the emissivity, which creates a ground level IR environment that is then partially absorbed as it travels upward through the atmosphere to the altitude of the balloon.

The environment is specified with:

a) Five-point Atmosphere model; temperature as a function of altitude which implies pressure

b) Winds aloft; wind speed and direction as a function of altitude

c) Launch location and month/day/time of launch

d) Day/night ground temperatures and emissivity as a function of time

e) Cloud altitude implying cloud temperature

f) Ground albedo as a function of time

g) Cloud albedo as a function of time

h) Cloud sky obscuration fraction a function of time

i) Sun elevation angle as a function of local time and latitude/longitude

The high-altitude scientific balloon is a thermal vehicle. It lives in a mostly radiant thermal balance from the many heat sources that influence it. When warm, the gas expands and either pressurizes the gas envelope or is vented. If vented as happens with a zero-pressure balloon, then nighttime cooling makes ballast drops necessary. Soon one runs out of both ballast and gas, which is why performance predictions are critical. Super-pressure balloon design also benefits, as the day/night pressure drop is a critical bit of information during the design process.

These are the basic heat sources that influence balloon flight:

a) Direct visible light sunshine on the skin membrane

b) Reflected diffuse sunshine in the form of planetary ground albedo

c) Reflected diffuse sunshine in the form of planetary cloud albedo

d) Diffuse infrared from the ground/atmosphere

e) Diffuse infrared from the clouds

f) Internal infrared "self-glow" of interior skin

g) Atmospheric convection

h) Diffuse infrared from the sky (important only in environments like Venus)

To give one an idea of the influences at 33 kilometers altitude on an average sunny day, the fraction of heat loads absorbed in the skin of a large scientific balloon (10 to 40 million cubic foot volume) is approximately:

Planetary surface IR $\quad 49 \%$

Direct solar $\quad 35 \%$

Indirect solar (albedo) $\quad 20 \%$

Self-glow internal IR $\quad 3 \%$

Atmospheric convection $\quad-7 \%$

\section{Theoretical Development}

I will not describe every equation in the program, but rather focus on some of the more unique or essential formulations.

\section{A. Approximate Geometric Properties}

Depending on the three classes of balloons treated in the software (spherical, zero pressure natural shape, or super-pressure pumpkin), geometric properties are approximated with the following equations. 
First, the unconstrained volume of the bubble, $\mathrm{m}^{3}$ :

$$
\text { Volume }=M_{\text {gas }} \cdot R_{\text {gas }} \cdot \frac{T_{g a s}}{P_{g a s}}
$$

Equations for a zero-pressure shape, closest match for a sigma shape $=0.2$ :

Balloon diameter, top view, $\mathrm{m}$ :

$$
\text { Diameter }=2.23 \cdot\left(\frac{0.75}{\pi} \cdot \frac{M_{\text {gas }}}{\rho_{\text {gas }}}\right)^{\frac{1}{3}}
$$

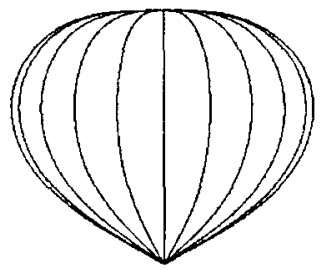

Length of gore exposed in the bubble, $\mathrm{m}$ :

$$
L_{\text {goreB }}=1.37 \cdot \text { Diameter }
$$

Surface area showing in a zero pressure shape:

$$
\begin{aligned}
A_{\text {surf }} & =2.582 \cdot \text { Diameter }^{2} \\
A_{\text {surf }} & =4.94 \cdot \text { Volume }^{\frac{2}{3}}
\end{aligned}
$$

Maximum "crenellated" surface area in a zero pressure shape not fully inflated:

$$
A_{\text {surf } 1}=4.94 \cdot \text { Volume }_{\text {design }} \frac{2}{3} \cdot\left(1-\cos \left(\pi \cdot L_{\text {gore B }} / L_{\text {gore Design }}\right)\right)
$$

To include the effect of the crenellated excess surface area for convection and film mass calculations, an estimate is made regarding the effective exposed surface area:

$$
A_{\text {effective }}=0.65 \cdot A_{\text {surf }}+0.35 \cdot A_{\text {surf } 1}
$$

Top projected area:

$$
A_{\text {top }}=\frac{\pi}{4} \cdot \text { Diameter }^{2}
$$

Height of balloon in a zero pressure shape:

$$
\text { Height }=0.748 \cdot \text { Diameter }
$$

Approximate average differential pressure due to buoyancy in a zero pressure design:

$$
\Delta P=g \cdot\left(\rho_{\text {air }}-\rho_{\text {gas }}\right) \cdot 0.834 \cdot\left[\frac{0.75}{\pi} \cdot \frac{M_{\text {gas }}}{\rho_{\text {gas }}}\right]^{\frac{1}{3}}
$$

\section{Equations for a super-pressure pumpkin shape}

Diameter, top view, m:

$$
\text { Diameter }=2.296 \cdot\left(\frac{0.75}{\pi} \cdot \frac{M_{\text {gas }}}{\rho_{\text {gas }}}\right)^{\frac{1}{3}}
$$

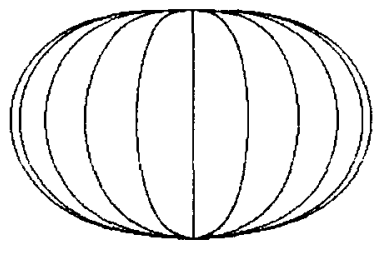


Length of gore in a pumpkin balloon, $\mathrm{m}$ :

$$
L_{\text {gore }}=1.312 \cdot \text { Diameter }
$$

Surface area showing in a pumpkin shape:

$$
\begin{aligned}
& A_{\text {surf }}=2.635 \cdot \text { Diameter }^{2} \\
& { }^{\text {or }} A_{\text {surf }}=5.3 \cdot \text { Volume }^{\frac{2}{3}}
\end{aligned}
$$

Height of balloon in a pumpkin shape:

$$
\text { Height }=0.604 \cdot \text { Diameter }
$$

Average differential pressure when in a super-pressure mode:

$$
\Delta P=\frac{M_{\text {gas }} \cdot R_{\text {gas }} \cdot T_{\text {gas }}}{\text { Volume }_{\text {design }}}-P_{\text {air }}
$$

If the unconstrained volume is greater than the maximum design volume Volume $_{\text {design }}$, then the required average gas pressure is recalculated. If the balloon is a zero pressure design, then the gas will flow out the ducts with the elevated pressure according to well-established duct-flow equations. If the balloon is a pumpkin super-pressure design, the differential pressure can continue to rise until equilibrium is established or a vent is opened.

$$
P_{\text {gas }}=P_{\text {air }}+\Delta P
$$

\section{Illuminated Projected Area}

The illuminated projected area of a balloon varies with solar elevation angle $E L V$, and uses the top projected area $A_{t o p}$ as the reference. Equation 16a was derived from information in Ref. 2.

For a zero pressure shape:

For a pumpkin shape:

For a spherical shape:

$$
\begin{aligned}
& A_{\text {projected }}=A_{\text {top }} *[0.9125+0.0875 \cdot \cos (\pi-2 \cdot E L V)] \\
& A_{\text {projected }}=A_{\text {top }} *[0.8219+0.1781 \cdot \cos (\pi-2 \cdot E L V)]
\end{aligned}
$$

\section{B. Atmospheric Transmissivity}

The transmissivity of optical frequency irradiance thru the atmosphere follows a Beer's Law format of exponential decay, the constants in the equation being derived experimentally for a clear atmosphere. A correction factor $C F_{\text {airmass }}$ is factored into the air mass ratio to account for fog and smoke, or for a different planet's atmosphere. This is a modification to the original equation found in Ref.1.

The Air mass ratio that the sun shines thru is calculated with the surface pressure $P_{0}:{ }^{1}$

$$
\text { AirMass }=C F_{\text {airmass }} \cdot\left(\frac{P_{\text {air }}}{P_{o}}\right) \cdot\left[\sqrt{1229+(614 \cdot \sin (E L V))^{2}}-614 \cdot \sin (E L V)\right]
$$

The transmissivity of a solar beam thru the atmosphere is then correlated with the air mass ratio: ${ }^{1}$

$$
\tau_{\text {atm }}=0.5 \cdot\left[e^{-0.65 \cdot \text { AirMass }}+e^{-0.95 \cdot \text { AirMass }}\right]
$$

For the situation when the sun elevation angle is below the true horizon but above the apparent horizon, use this approximate formula to determine the air mass ratio (see Fig. 1):

$$
\text { AirMass }=\frac{P_{\text {air }}}{P_{o}} \cdot\left[1+\frac{E L V}{\operatorname{Dip}}\right]-70 \cdot \frac{E L V}{\text { Dip }}
$$




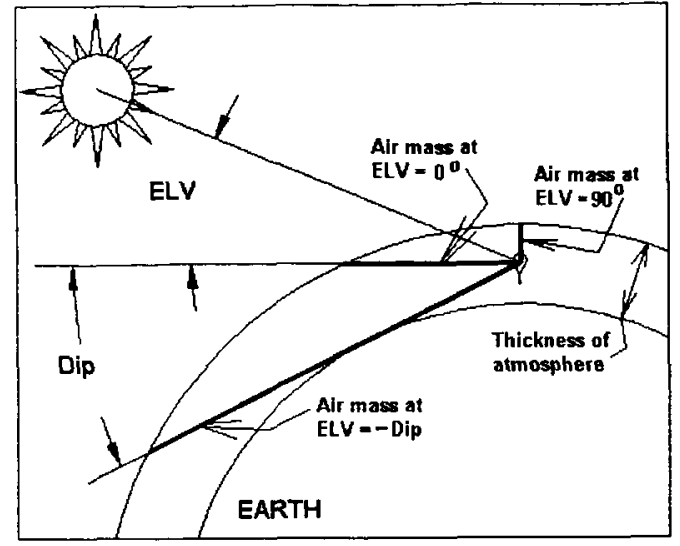

Figure 1. Dip of the horizon and air mass.

\section{Direct Solar Environment}

To generalize the solar environment for any planet, we must know the orbital eccentricity $e$ and the mean orbital radius $R_{A U}$. For small orbital eccentricities, one can approximate the true anomaly $T A$ as being reasonably close to the value of the much easier to calculate mean anomaly $M A$ :

$$
T A \approx M A=2 \pi \cdot \frac{\text { Day }_{\text {number }}}{\text { DaysPerYear }}
$$

The day number begins at perihelion (closest and hottest).

$$
I_{S u n}=\frac{1358}{R_{A U}{ }^{2}} \cdot\left[1+\frac{1}{2} \cdot\left[\left\{\frac{1+e}{1-e}\right\}^{2}-1\right] \cdot \cos (T A)\right] \mathrm{Watts} / \mathrm{m}^{2}
$$

For Earth, $R_{A U}=1, e=0.016708$, for Mars, $R_{A U}=1.52368, e=0.093400$, and for Venus, $R_{A U}=0.723, e=0.0068$

At the balloon altitude $Z$, the direct solar irradiance is equal to the product of the top of the atmosphere value and the atmospheric transmittance:

$$
I_{\text {SunZ }}=I_{\text {Sun }} \cdot \tau_{\text {atm }}
$$

\section{Infrared environment}

Just as direct solar is attenuated for thickness of the atmosphere, the same is applied to ground and cloud IR that has to pass through a certain amount of atmosphere to reach the balloon. It is ASSUMED that the same coefficients in the equations from section C. apply to IR as well, even though there absorption lines for $\mathrm{H}_{2} \mathrm{O}$ and $\mathrm{CO}_{2}$.

Infrared diffuse flux at ground level with ground emissivity $\varepsilon_{\text {ground }}$ and ground temperature $T_{\text {ground }}\left({ }^{\circ} \mathrm{K}\right)$ :

$$
q_{\text {IRground }}=\varepsilon_{\text {ground }} \cdot \sigma \cdot T_{\text {ground }}{ }^{4} \quad \text { Watts } / \mathrm{m}^{2}
$$

As with visible light, the ground IR is attenuated with a similar formula to Eq. (18):

$$
\tau_{\text {atmlR }}=1.716-0.5 \cdot\left[e^{-0.65 \cdot \frac{P_{\text {air }}}{P_{o}}}+e^{-0.95 \cdot \frac{P_{\text {air }}}{P_{o}}}\right]
$$

The ground-source diffuse IR flux at the balloon altitude $Z$ :

$$
q_{I R g r o u n d ~}=q_{I R g r o u n d} \cdot \tau_{\text {atm IR }} \quad \text { Watts } / \mathrm{m}^{2}
$$

Deserts will have the greatest day/night temperature swings due to little water acting as heat capacity/storage. Somewhere on the order of $20^{\circ} \mathrm{C}$ swing is not unreasonable. Oceans should have the least day/night swing in surface temperature. In the software, BalloonAscent will use a user-defined maximum day and a minimum night temperature and sine wave between the two with a user-defined lag (in fractions of a day, 0.05 default).

Here is a suggested list of ground emissivity:

Desert; $\varepsilon_{\text {ground }}=0.85$ (data suggests great variation within a region, as low as 0.8 )

Average ground; $\varepsilon_{\text {ground }}=0.95$

Snow; $\varepsilon_{\text {ground }}=0.98$

It is fortuitous that in the hottest places, the emissivity is low and in the coldest places the emissivity is high. 


\section{E. Albedo Environment}

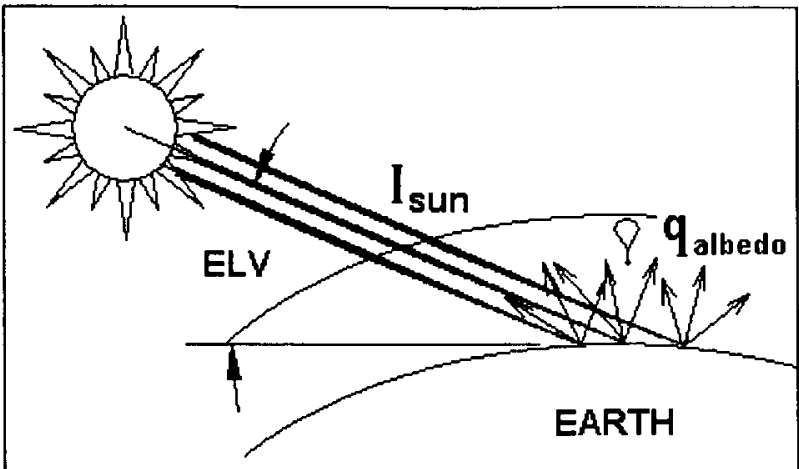

Figure 2. Albedo model at the top of the atmosphere

There is a simple model relating surface albedo and albedo flux. The spacecraft EOS-TERRA has data on shortwave radiation flux average values and the calculated planetary albedo. The numbers are averaged monthly and so must include normal average cloud cover. The assumption here is that the albedo number is the total specular + diffuse solar reflection, but treated as diffuse. BalloonAscent uses the effect of solar elevation angle to achieve the instantaneous time varying albedo flux. One can see from Fig. 2 that the incoming beam of sunlight of flux strength Isun is diminished on its transmission to the ground thru the atmosphere. Since the solar angle is not directly overhead, the beam of light is smeared out over a larger surface area, diminishing it further by the factor of $\sin (E L V)$. The measurements from spacecraft are from the top of the atmosphere, so this model under calculates for when the balloon is at low altitude. It is further assumed that atmospheric glow is part of the measured number.

Albedo flux at the top of the atmosphere:

$$
q_{\text {albedo }}=\text { Albedo } \cdot I_{\text {Sun }} \cdot \sin (E L V) \text { Watts } / \mathrm{m}^{2}
$$

\section{F. Cloud Modifications}

The cloud fraction $C F$ is defined as the fraction of the sky that is totally obscured by clouds. Note that clouds attenuate the direct solar when the balloon is under the cloud level. Planetary albedo is attenuated differently when below due to a one pass thru the clouds effect, and two passes through the clouds when the balloon is above the clouds. Earth IR is attenuated when above the cloud layer due to obscuration. When below the cloud level, there is no effect due to cloud albedo, but cloud albedo is counted only when above the cloud level.

Solar beam radiation modified for clouds:

If the altitude $Z$ is below the clouds then direct solar flux $\quad q_{S u n}=I_{\text {SunZ }} \cdot(1-C F)$ watts $/ \mathrm{m}^{2}$

If the altitude $Z$ is above the clouds then direct solar flux $\quad$ qsun $=I_{\text {SunZ }}$

Planetary diffuse IR radiation modified for clouds:

If the altitude $Z$ is below the clouds then $q_{I R p l a n e t}=q_{I R g r o u n d}+C F \cdot q_{I R c t o u d Z} \quad$ watts $/ \mathrm{m}^{2}$

If altitude $Z$ above clouds then

$$
q_{I R p l a n e t}=q_{I R g r o u n d Z} \cdot(1-C F)+C F \cdot q_{I R c l o u d Z}
$$

Albedo modified for clouds:

If the altitude $Z$ is below the clouds then $A l b e d o=A l b e d o_{\text {Ground }} \cdot(1-C F) \quad$ watts $/ \mathrm{m}^{2}$

If altitude $Z$ above clouds then

$$
\text { Albedo }=\text { Albedo }_{\text {Ground }} \cdot(1-C F)^{2}+\text { Albedo }_{\text {Cloud }} \cdot C F
$$

\section{G. Averaging the Film Optical Properties}

The optical surface properties are expressed as absorptivity $\alpha$, transmissivity $\tau$, and emissivity $\varepsilon$. The balloon surface is treated as an area-average between the properties of the balloon top (cap + shell) and the bottom (shell only), and then area-averaged again with the properties of the meridional load tapes.

The view factor ViewFactor is the balloon surface area diffuse-radiant view factor of the planet. It is the ratio of balloon surface area that "sees" the planet surface divided by the total exposed balloon surface area. $R_{\text {planet }}$ is the radius of the planet, meters, and is equal to 6371000 for earth.

$$
\text { HalfCone }_{\text {angle }}=\sin ^{-1}\left[\frac{R_{\text {Planet }}}{R_{\text {Planet }}+Z}\right]
$$




$$
\text { ViewFactor }=\frac{\left(1-\cos \left(\text { HalfCone }_{\text {angle }_{1}}\right)\right)}{2}
$$

\section{H. Radiant Heat Loads on the Film}

The following equations will relate the radiant fluxes affecting the balloon skin, and the resulting absorbed

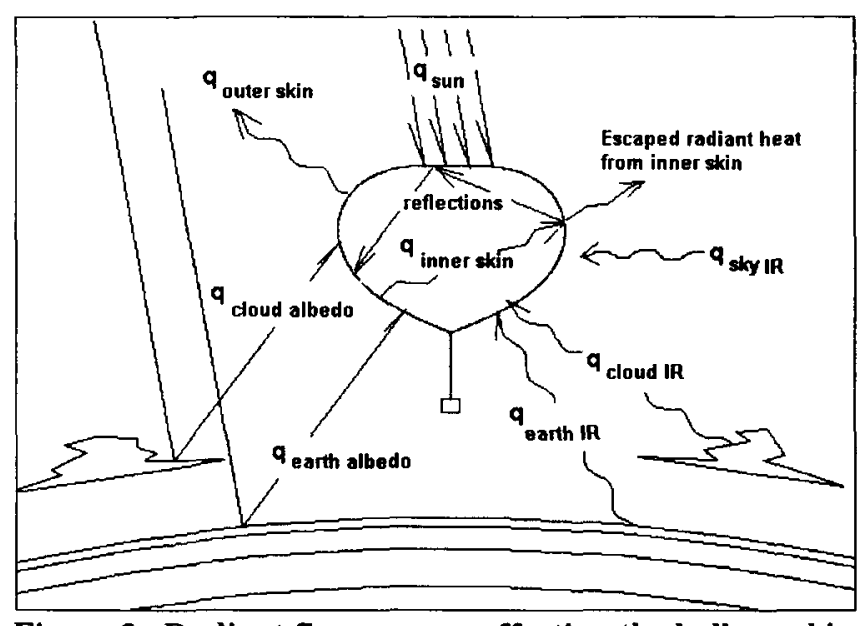

Figure 3. Radiant flux sources affecting the balloon skin radiant heat loads. As can be seen in Fig.3, the film is not only affected by the outer environment, but by the inner environment and the trapping of heat by multiple reflections. These multiple reflections raise the effective interior absorption. Recalling that reflectivity $r=1-\alpha-\tau$, and the effective reflectivity becomes $r_{\text {effective }}=\mathrm{r}+\mathrm{r}^{2}+\mathrm{r}^{3}+\mathrm{r}^{4}+\mathrm{r}^{5}+\ldots$

From Kirchoff's law of radiation heat transfer, at any specific wavelength the absorptivity is equal to the emissivity while in thermal equilibrium. Even though this analysis is for transient conditions, we still follow this rule. This is important because absorptivity is generally given for solar short-wave frequencies, but emissivity is given for IR wavelengths. So if we are interested in the absorption of IR radiant energy, we use the given IR emissivity as our IR absorptivity ( $\alpha_{I R}$ $=\varepsilon)$.

Absorbed direct sunlight heat:

$$
Q_{\text {Sun }}=\alpha \cdot A_{\text {projected }} \cdot q_{\text {Sun }} \cdot\left[1+\tau \cdot\left(1+r_{\text {effective }}\right)\right] \quad \text { Watts }
$$

Absorbed albedo heat:

$$
Q_{\text {Albedo }}=\alpha \cdot A_{\text {surf }} \cdot q_{\text {Albedo }} \cdot \text { ViewFactor } \cdot\left[1+\tau \cdot\left(1+r_{\text {effective }}\right)\right] \quad \text { Watts }
$$

Absorbed planetary IR heat:

$$
Q_{\text {IRplanet }}=\alpha_{I R} \cdot A_{\text {surf }} \cdot q_{\text {IRplanet }} \cdot \text { ViewFactor } \cdot\left[1+\tau_{I R} \cdot\left(1+r_{\text {effective }}\right)\right] \quad \text { Watts }
$$

Absorbed IR from the sky:

$$
Q_{I R s k y}=\alpha_{I R} \cdot A_{\text {surf }} \cdot q_{I R s k y} \cdot(1-\text { ViewFactor }) \cdot\left[1+\tau_{I R} \cdot\left(1+r_{\text {effective }}\right)\right] \quad \text { Watts }
$$

Absorbed IR self-glow from the interior:

$$
Q_{\text {IRfilm }}=\sigma \cdot \varepsilon \cdot \alpha_{I R} \cdot A_{\text {surf }} \cdot T_{\text {film }}{ }^{4} \cdot\left(1+r_{\text {effective }}\right) \quad \text { Watts }
$$

Emitted IR energy from both interior and exterior of the balloon skin:

$$
Q_{\text {IRout }}=\sigma \cdot \varepsilon \cdot 2 \cdot A_{\text {surf }} \cdot T_{\text {film }}{ }^{4} \quad \text { Watts }
$$

\section{Convective Heat Loads on the Film}

The convective heat transfer of the balloon involves transfers between the atmosphere and the exterior skin, and the lifting gas with the interior skin. The external convection is partitioned into free convection and forced convection.

\section{Air and Gas Properties}


Dynamic viscosity of air $^{3}$ :

$$
\mu_{\text {air }}=\frac{1.458 \cdot 10^{-6} \cdot T_{\text {air }}{ }^{1.5}}{T_{\text {air }}+110.4} \quad \mathrm{~N}-\mathrm{s} / \mathrm{m}^{2}
$$

Dynamic viscosity of helium gas ${ }^{3}$ :

$$
\mu_{\text {gas }}=1.895 \cdot 10^{-5} \cdot\left(\frac{T_{\text {gas }}}{273.15}\right)^{0.647} \quad \mathrm{~N}-\mathrm{s} / \mathrm{m}^{2}
$$

Conductivity of air ${ }^{3}$ :

$$
k_{\text {air }}=0.0241 \cdot\left(\frac{T_{\text {air }}}{273.15}\right)^{0.9}
$$

Watts $/ \mathrm{m}-{ }^{\circ} \mathrm{K}$

Conductivity of helium gas ${ }^{3}$ :

$$
k_{g a s}=0.144 \cdot\left(\frac{T_{g a s}}{273.15}\right)^{0.7}
$$

Prandtl number for air, dimensionless ${ }^{3}$ :

$$
\operatorname{Pr}_{\text {air }}=0.804-3.25 \cdot 10^{-4} \cdot T_{\text {air }}
$$

Prandtl number for helium gas, dimensionless ${ }^{3}$ :

$$
P r_{\text {gas }}=0.729-1.6 \cdot 10^{-4} \cdot T_{\text {gas }}
$$

\section{External Free Convection}

Dimensionless Grashof number:

$$
G r_{\text {air }}=\frac{\rho_{\text {air }}{ }^{2} \cdot g \cdot\left|T_{\text {fim }}-T_{\text {air }}\right| \cdot \text { Diameter }^{3}}{T_{\text {air }} \cdot \mu_{\text {air }}^{2}}
$$

Dimensionless Nusselt number for a sphere in free convection:'

$$
N u_{\text {air }}=2+0.45 \cdot\left(G r_{\text {air }} \cdot P r_{a i r}\right)^{0.25}
$$

Free convection heat transfer coefficient for the external skin:

$$
H C_{\text {free }}=\frac{N u_{\text {air }} \cdot k_{\text {air }}}{\text { Diameter }} \quad \text { Watts } / \mathrm{m}^{2}-{ }^{\circ} \mathrm{K}
$$

\section{External Forced Convection}

When the balloon is ascending, the convection does not rely on differentially warm buoyant air for convection, but rather the vertical velocity forces the relative movement of air. First we calculate the Reynolds number, which is the dimensionless ratio of inertial forces to viscous forces; $R e=V e l o c i t y \cdot$ Diameter $\cdot \rho_{\text {air }} / \mu_{\text {air }}$

Forced convection heat transfer coefficient for the external skin: ${ }^{3}$

$$
H C_{\text {forced }}=\frac{k_{\text {air }}}{\text { Diameter }} \cdot\left(2+0.41 \cdot \operatorname{Re}^{0.55}\right) \quad \text { Watts } / \mathrm{m}^{2}-{ }^{\circ} \mathrm{K}
$$

\section{Internal Free Convection}

Reference 3 has an equation for the heat transfer coefficient inside a sphere, which BalloonAscent uses unaltered, and is reproduced here: 


$$
H C_{\text {intemal }}=0.13 \cdot k_{\text {gas }} \cdot\left(\frac{\rho_{g a s}{ }^{2} \cdot g \cdot\left|T_{\text {film }}-T_{\text {gas }}\right| \cdot P r_{g a s}}{T_{\text {gas }} \cdot \mu_{\text {air }}{ }^{2}}\right)^{\frac{1}{3}} \quad \text { Watts } / \mathrm{m}^{2}-\mathrm{K}^{\mathrm{K}}
$$

\section{Total Convective Heat Transfer}

For the external heat convection, the greater of the two values between free and forced convection is selected to equal $H C_{\text {external. }}$. The heat loads are thus:

External convection heat load: $\quad Q_{\text {ConvExt }}=H C_{\text {external }} \cdot A_{\text {effective }} \cdot\left(T_{\text {air }}-T_{\text {film }}\right) \quad$ Watts

Internal convection heat loss: $\quad Q_{\text {ConvInt }}=H C_{\text {intermal }} \cdot A_{\text {effective }} \cdot\left(T_{\text {film }}-T_{\text {gas }}\right) \quad$ Watts

\section{J. Lifting Gas Temperature Differential Equation}

The lifting gas rate of change of temperature is formulated on the adiabatic expansion response modified with internal convection interaction with the film. Whether from mass loss or purely a change of volume, or a combination of both, the gas will respond according to adiabatic expansion if the heat input $Q_{\text {ConvInt }}$ is zero.

$$
\frac{d T_{\text {gas }}}{d t}=\frac{Q_{\text {ConvInt }}}{c_{v} \cdot M_{\text {gas }}}+(\gamma-1) \cdot \frac{T_{g a s}}{\rho_{\text {gas }}} \cdot \frac{d \rho_{\text {gas }}}{d t}
$$

Expressed in terms of mass and volume change derivatives:

$$
\frac{d T_{\text {gas }}}{d t}=\frac{Q_{\text {Convint }}}{M_{\text {gas }} \cdot c_{v}}+(\gamma-1) \cdot T_{\text {gas }} \cdot\left(\frac{d M_{\text {gas }}}{d t} \cdot \frac{1}{M_{\text {gas }}}-\frac{d \text { Volume }}{d t} \cdot \frac{1}{\text { Volume }}\right) \quad{ }^{\circ} \mathrm{K} / \mathrm{s}
$$

Where $\gamma=c_{p} / c_{v}$, and $R_{\text {gas }} / c_{\nu}=\gamma-1$

\section{K. Film Temperature Differential Equation}

The rate of change of the film temperature is derived from the simple transient energy-balance equation:

$$
\frac{d T_{\text {film }}}{d t}=\frac{\left(Q_{\text {Sun }}+Q_{\text {Albedo }}+Q_{\text {IRplanet }}+Q_{\text {IRsky }}+Q_{\text {IRfilm }}+Q_{\text {ConvExt }}-Q_{\text {ConvIm }}-Q_{\text {IRout }}\right)}{c_{f} \cdot M_{\text {fllm } B}} \quad{ }^{\circ} \mathrm{K} / \mathrm{s}
$$

\section{Valve and Duct Flow Differential Equations}

Flow thru a duct or valve depends on the differential pressure across the area interface, the cross sectional area, the density of the gas, and a discharge coefficient. The velocity thru such a duct or valve is:

$$
\text { Velocity }_{\text {flow }}=C_{\text {discharge }} \cdot \sqrt{2 \cdot \frac{\Delta P_{\text {valve }}}{\rho_{\text {gas }}}} \quad \mathrm{m} / \mathrm{s}
$$

This leads to the differential equation:

$$
\frac{d M_{\text {gas }}}{d t}=-A_{\text {valve }} \cdot C_{\text {discharge }} \cdot \sqrt{2 \cdot \Delta P_{\text {valve }} \cdot \rho_{\text {gas }}} \quad \mathrm{kg} / \mathrm{s}
$$

The mass flow can apply to either a duct flow situation as in a zero pressure balloon, or a valving situation as in a pressurized balloon. 


\section{3-D Acceleration Differential Equations}

The total buoyant force produced by the balloon is the gross inflation, GI. With the average differential pressure and the gas temperature, the gas density is determined with the ideal gas law. The ambient air density is known, and with this information the specific buoyancy $b$ is determined:

$$
b=g \cdot\left(\rho_{\text {air }}-\rho_{\text {gas }}\right) \quad \mathrm{N} / \mathrm{m}^{3}
$$

The gross inflation:

$$
G I=b \cdot \text { Volume } \quad \mathrm{N}
$$

The aerodynamic forces are determined from the relative wind with respect to the balloon's motion. If $U x, U y, U z$ are the balloon's absolute velocity components, and $V$ wind $_{x}, V$ wind $_{y}, V$ wind $_{Z}$ are the wind's absolute velocity, then the following are the relative velocities:

$$
\begin{aligned}
& \text { Vrelative }_{X}=\text { Vwind }_{X}-U_{X} \\
& \text { Vrelative }_{Y}=\text { Vwind }_{Y}-U_{Y} \\
& \text { Vrelative }_{Z}=\text { Vwind }_{Z}-U_{Z}
\end{aligned}
$$

The magnitude of the relative wind:

$$
\text { Vrelative }=\left(\text { Vrelative }_{X}{ }^{2}+\text { Vrelative }_{Y}{ }^{2}+\text { Vrelative }_{Z}{ }^{2}\right)^{\frac{1}{2}} \quad \mathrm{~m} / \mathrm{s}
$$

The magnitude of the drag force, where $C_{d}$ is the drag coefficient and the reference area is the top projected area:

$$
\operatorname{Drag}=\frac{1}{2} \cdot \rho_{\text {air }} \cdot \text { Vrelative }{ }^{2} \cdot C_{d} \cdot A_{\text {top }} \quad \mathrm{N}
$$

The drag coefficient has in the literature for a sphere values ranging from 0.1 to 0.47 . However, a rising balloon is not a sphere, and more resembles an ice-cream cone which may have an equivalent drag coefficient based on the calculated spherical diameter of $C_{d} \approx 0.8$. The drag is now partitioned into its vector components:

$$
\begin{aligned}
& \text { Drag }_{X}=\text { Drag } \cdot \text { Vrelative }_{X} / \text { Vrelative } \\
& \text { Drag }_{Y}=\text { Drag } \cdot \text { Vrelative }_{Y} / \text { Vrelative } \\
& \text { Drag }_{Z}=\text { Drag } \cdot \text { Vrelative }_{Z} / \text { Vrelative }
\end{aligned}
$$

The differential equation governing the vertical motion of the balloon where $M_{\text {gross }}$ is the over-all mass of the balloon, less gas (suspended mass plus balloon material mass):

$$
\frac{d U_{Z}}{d t}=\frac{G I+\operatorname{Drag}_{Z}-M_{\text {gross }} \cdot g}{M_{\text {virtual }}} \quad \mathrm{m} / \mathrm{s} / \mathrm{s}
$$

The virtual mass is calculated with a virtual mass coefficient $C_{\text {virual. }}$. This is to take into account the mass of air that is necessarily dragged along with the balloon mass. Values assumed range from $0.25<C_{\text {virtual }}<0.5$.

$$
M_{\text {virtual }}=M_{\text {gross }}+M_{\text {gas }}+C_{\text {virual }} \cdot\left(\rho_{\text {air }} \cdot \text { Volume }\right) \quad \mathrm{kg}
$$

The horizontal accelerations:

$$
\frac{d U_{X}}{d t}=\frac{\operatorname{Drag}_{X}}{M_{\text {virual }}} \quad \frac{d U_{Y}}{d t}=\frac{\operatorname{Drag}_{Y}}{M_{\text {virtual }}}
$$




\section{Software Interface}

The BalloonAscent interface is simple and intuitive. Figure 4 shows the main form in the background with a top row menu bar, which includes some handy balloon flight calculators. The sandwiched form is the data page, and it is subdivided into 13 categories, each accessible by clicking on the appropriately labeled command button. The data fields are made visible, sometimes with a new pop-up form, as is shown in the ambient environment data card. On the top row menu bar there are location templates to load coordinates and dates from the typical NSBF launch sites, as well as templates for typical NSBF zero pressure balloons. The ambient environment is defined such that it can vary four different times during the simulation. The maximum daytime and minimum nighttime temperature for a given area is defined, with a sine-wave variation between the two implemented automatically. The atmosphere can also be modified with a diurnal delta temperature variation and lag time, which will effectively compress the atmosphere slightly at night. The ground emissivity and albedo is defined, as well as the cloud albedo and cloud fraction, which just about sums up the typical float environment. As a bonus, which also helps to model off-world situations, the radiant flux environment (solar, albedo, IR up-welling, IR down-welling) can be directly defined using Beer's law exponential decay coefficients. The software has a calculator on the ambient environment data page to calculate the coefficients from planetary data that you might happen to have.

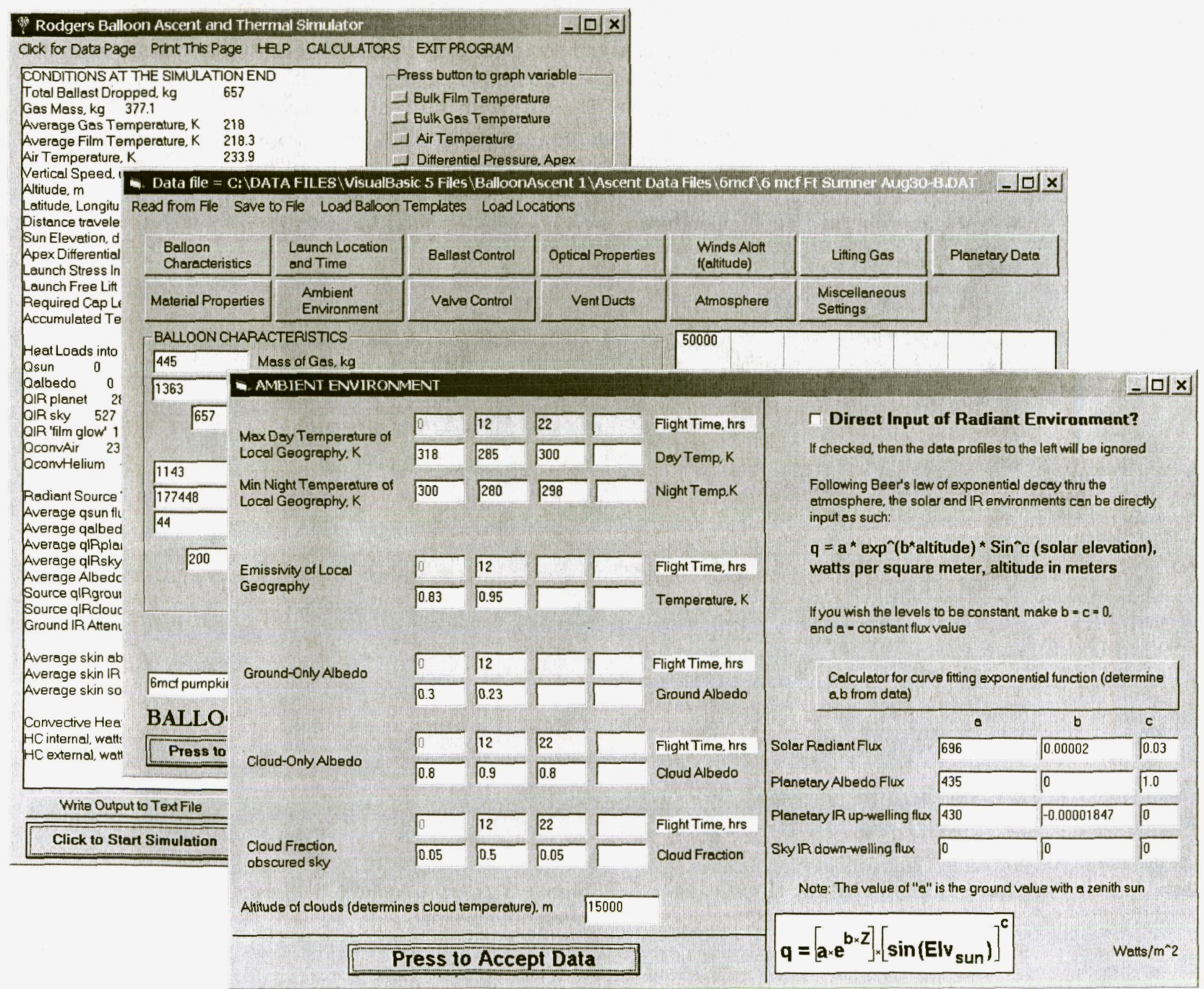

Figure 4. Program $1^{\text {st }}$ form, main data page, and ambient environment data card 


\section{Example Results}

\section{A. Adiabatic Test Runs}

To test whether or not the simulation was correctly determining the gas temperature during ascent or during a period of venting, the internal convective heat input to the gas $Q_{\text {Convm }}$ was set to zero. One would then expect the gas to behave in an adiabatic fashion according to the following equation:

$$
T_{2}=T_{1} \cdot\left(\frac{\rho_{2}}{\rho_{1}}\right)^{\gamma-1}
$$

Several runs were set up, the first an ascent with no venting, the second a pressurized pumpkin venting off pressure at a constant altitude. In both cases BalloonAscent followed the adiabatic expansion Eq. (56) exactly.

Considering that helium is a mono-atomic gas (no rotational or vibratory degrees of freedom) with weak Van de Walls forces when warm, it should follow the ideal gas laws assumed here quite well. Expansion thru small nozzles, where Thompson-Joule effects will deviate temperatures greatly from adiabatic assumptions, is not credibly applicable to ballooning except in situations during filling from helium bottles. This software does not address that portion of balloon operations, nor is it necessary for determining ascent or float behavior.

\section{B. Zero Pressure Balloon Simulation}

The 40 "heavy" zero-pressure balloon example was simulated here for a flight from McMurdo station Antarctica. Such balloons have seen diurnal variations in altitude on the order of 4500 meters, where as the simulation shows a larger variation on the order of 8000 meters (see Fig. 5). The change in altitude is caused by the variance in albedo flux, as can be seen in Fig. 6. The most logical explanation is that snow and ice have specular as well as diffuse albedo components, which will add complications to the simple diffuse albedo model of Eq. (26). Future versions will therefore include a specular albedo flux as a function of solar elevation angle and zenith surface reflectivity.

Figure 7 shows the gas, film, and air temperatures, and Fig. 8 is a ground track on an orthographic projection centered over the average location.

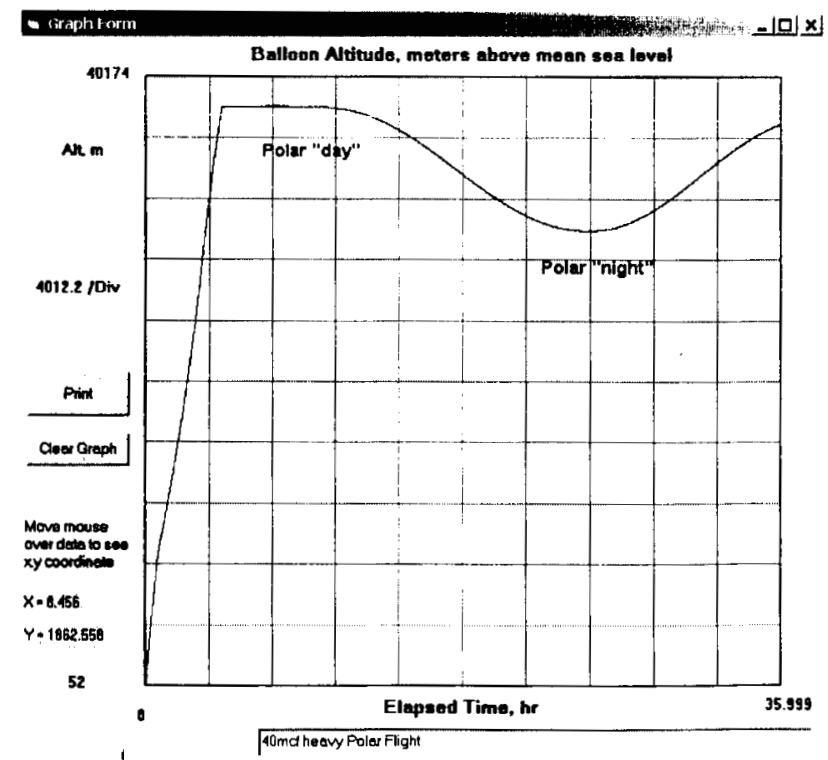

Figure 5. Zero pressure balloon altitude simulation

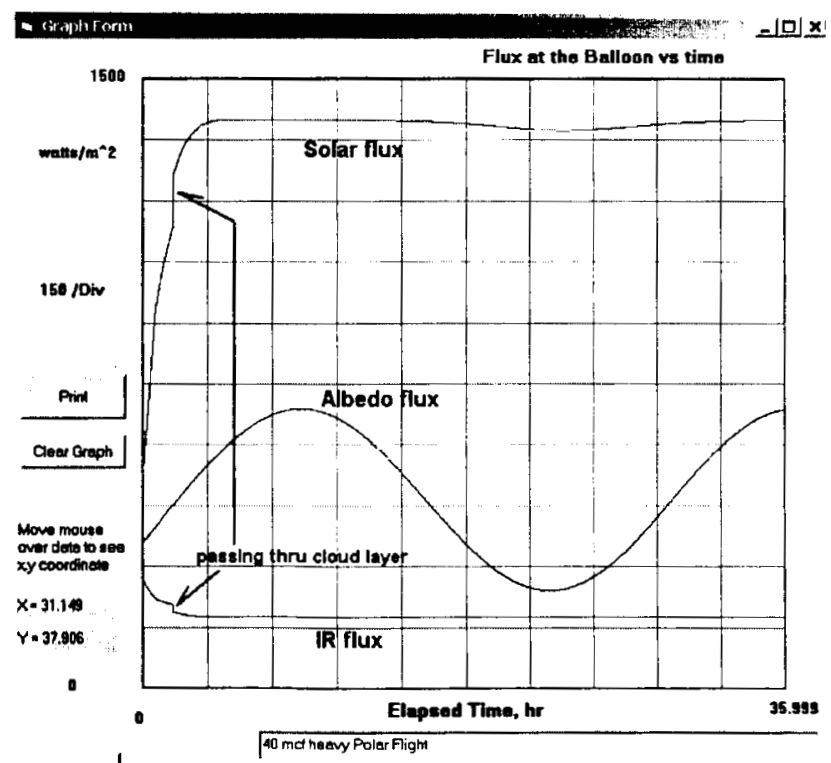

Figure 6. Zero pressure balloon environmental flux levels 


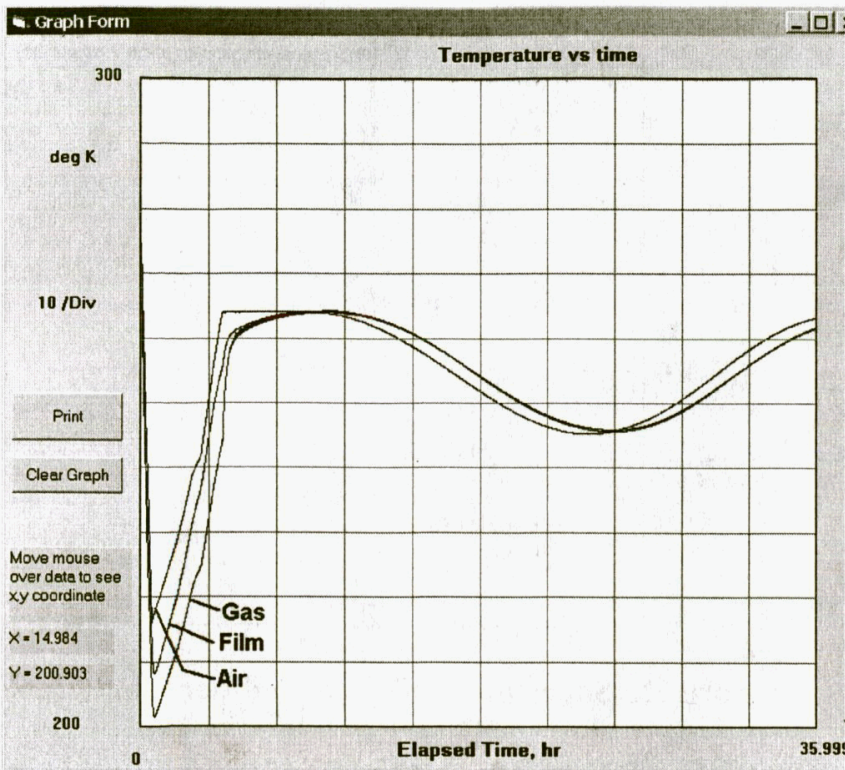

Figure 7. Zero pressure balloon temperatures

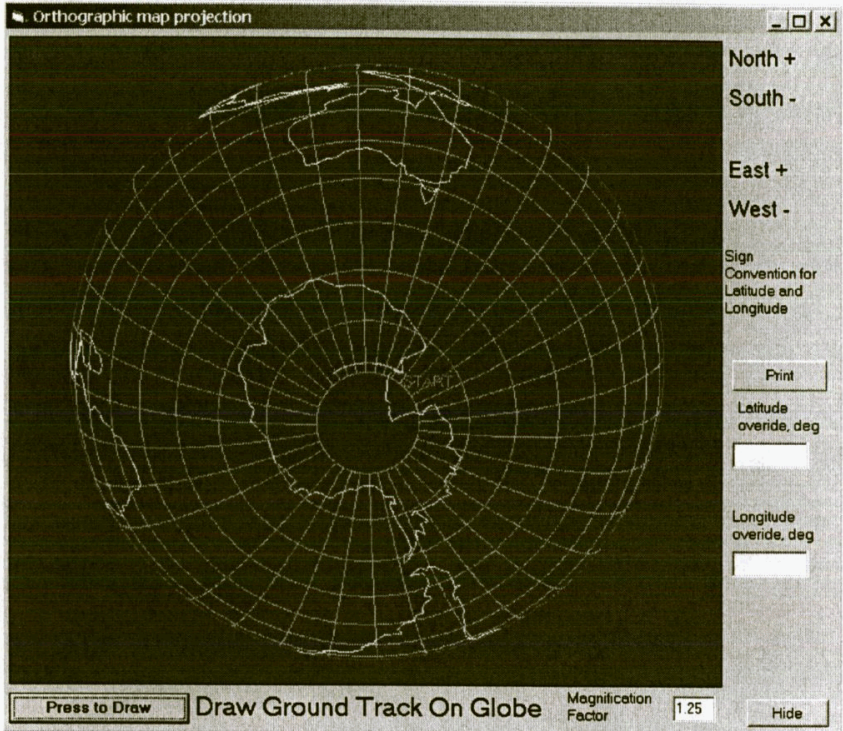

Figure 8. Zero pressure balloon ground track

\section{Super-Pressure Balloon Simulations}

The super-pressure example simulation is a 13 million cubic foot pumpkin with 245 gores, launched from Alice Springs Australia. It simulates the hot day case of traveling over the desert, and a cold case that night over a cold storm in the Pacific Ocean followed the next day by a typical mid-latitude warm ocean day. Figure 9 shows the variation in altitude; indeed the balloon looses all super pressure during the cold storm night and levels out at a lower altitude. Ballast could have been dropped, but to achieve 100 days of flight, an occasional altitude drop if not too severe is considered acceptable.

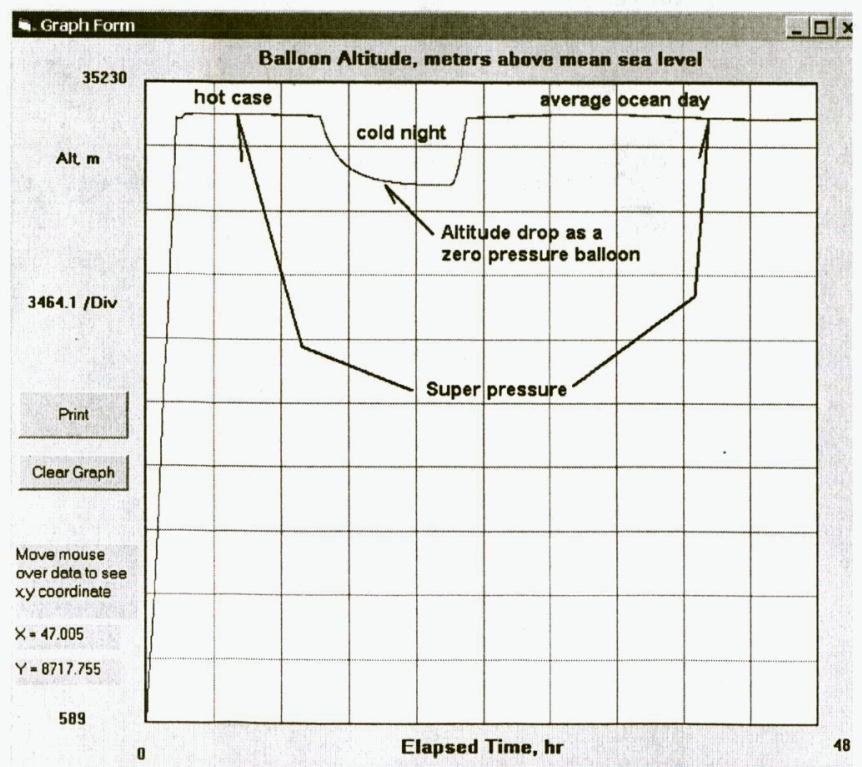

Figure 9. Super pressure balloon altitude variation

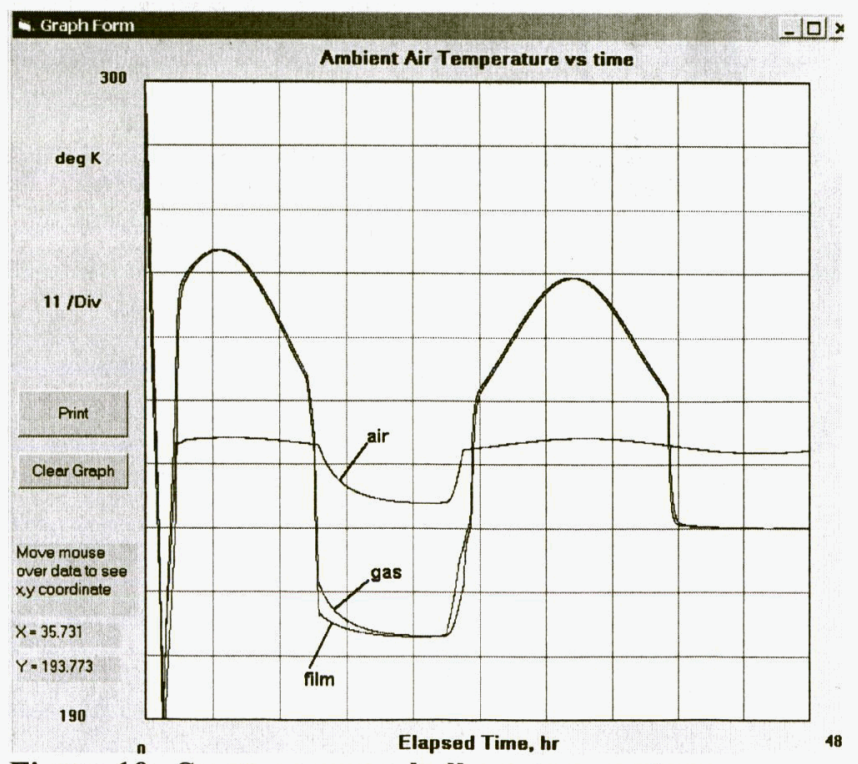

Figure 10. Super pressure balloon temperature variation 


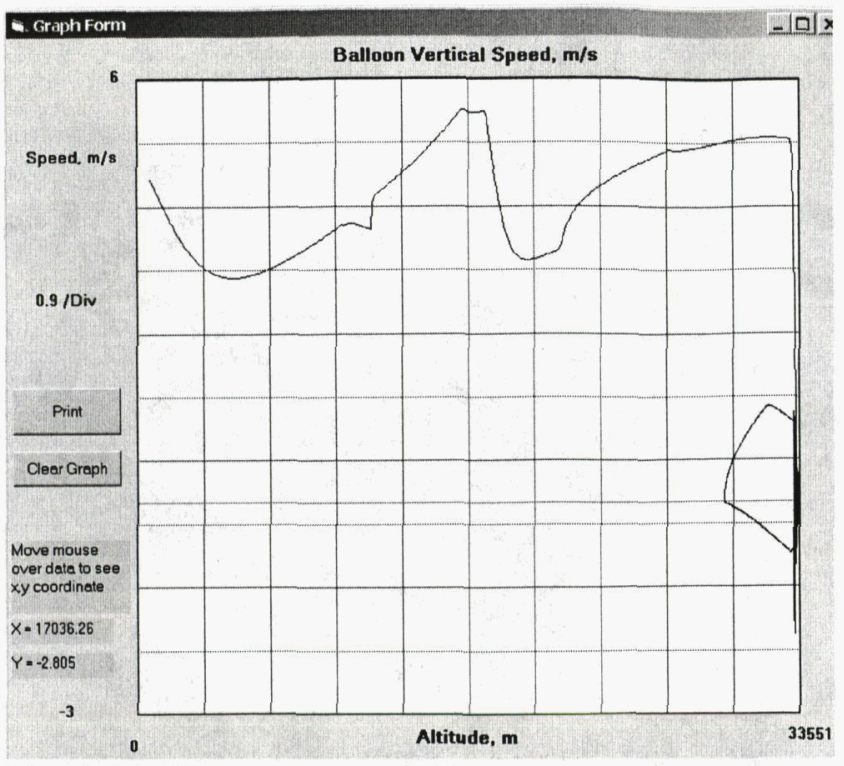

Figure 11. Super pressure balloon ascent velocity

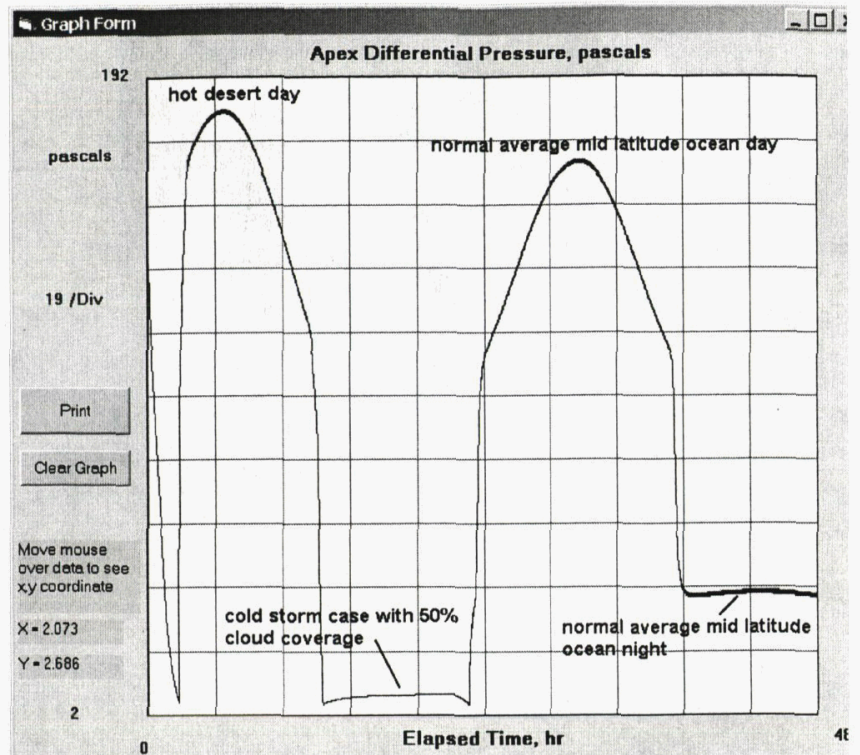

Figure 12. Super pressure balloon differential pressure

\section{Mars Balloon Simulation}

The two things to understand about Mars balloons are that scale-wise they fly at the bottom of the atmosphere. The atmosphere is thin, so the bottom offers the most buoyancy. This also forces the design payload to be small compared to the mass of the balloon material. The other consideration is the large diurnal temperature variation of the surface and atmosphere, reportedly $30^{\circ} \mathrm{K}$. This has the effect of compressing down the atmosphere at night, which leads to a very curious phenomenon regarding balloon flight on Mars if using a super-pressure design. The idea of the pressurized balloon is that so long as some internal pressure is maintained, the displaced volume is a constant. This means that the system density (total mass including gas $\div$ total displaced volume) is constant, and as all balloons, will match the atmospheric density when in float equilibrium. A Mars super-pressure balloon will find itself at night lighter than the surrounding air that has compressed down, and as a result will float upwards at night, and sink back down during the day, as shown in Fig. 13. Figure 14 shows the resulting radiant flux environment at the balloon's altitude.

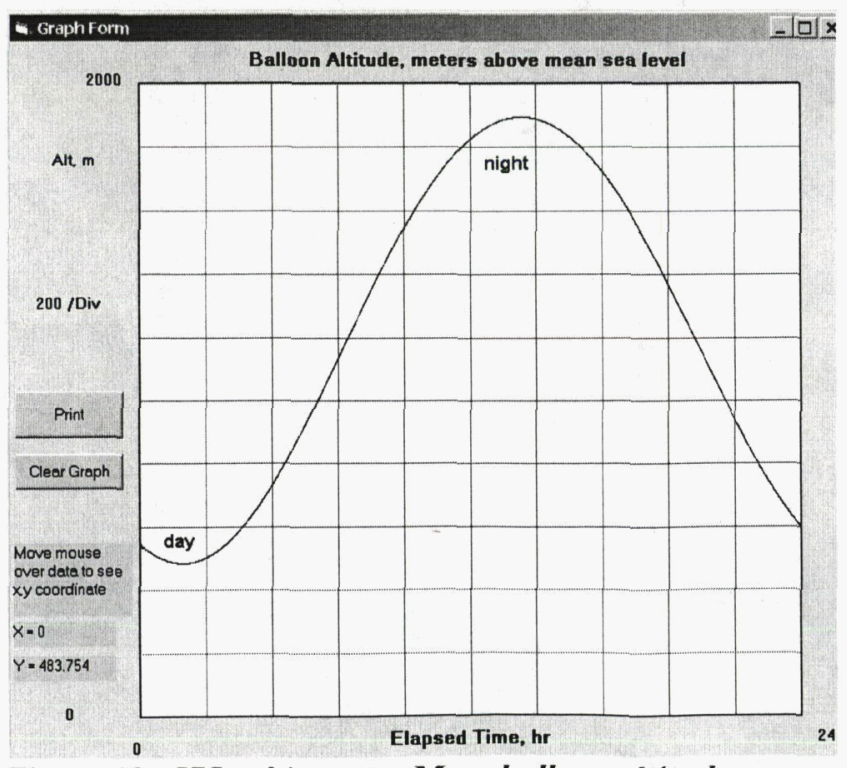

Figure 13. 575 cubic meter Mars balloon altitude

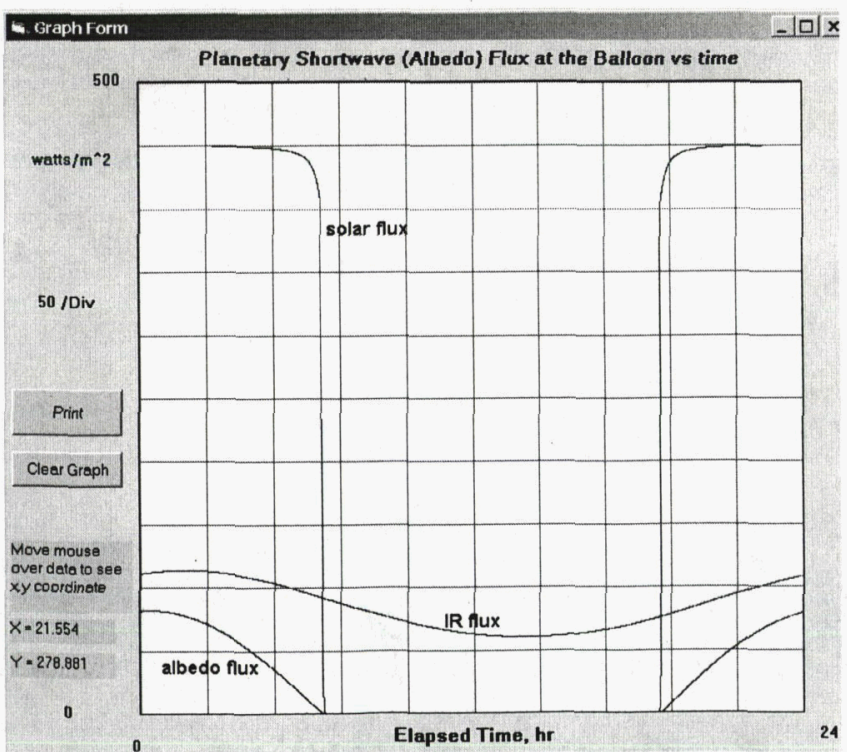

Figure 14. 575 cubic meter Mars balloon environment 


\section{Conclusion}

BalloonAscent is on its $32^{\text {nd }}$ version and still evolving. It is extremely user-friendly with intuitive data entry interfaces. The environment is defined with measurable parameters, and transmission models are used to propagate the environment to the balloon's altitude. Envisioned improvements in the future are to have built-in sigma shapes such that stub-ducts can be correctly modeled. A stub-duct is a short duct that is opened on command with a squib in order to lose a specific amount of gas such that a specific amount of altitude is lost. The other improvement has been mentioned already, to improve the albedo model for polar flights by including a specular component to the model (currently only diffuse is assumed). Another improvement is to consider a simple down-welling sky IR model as a function of altitude, which could add as much as 160 watts $/ \mathrm{m}^{2}$ at the launch altitude. This effect is admittedly very small, and at float the effect is nil.

\section{Acknowledgments}

I would like to thank Henry Cathey, Gabriel Garde, and Tracy Bohaboj of PSL, Danny Ball of NSBF, Mike Smith of Aerostar, Jim Rand of Winzen, and Debora Fairbrother of NASA for their inspiration and help in this effort.

\section{References}

${ }^{1}$ Kreith, F., and Kreider, J., Principles of Solar Engineering, Hemisphere Publishing Corp., 1978, Chap. 3.

${ }^{2}$ Cathey, H. M. "Transient Thermal Loading of Natural Shaped Balloons," AIAA 97-1498, 1997.

${ }^{3}$ Morris, A., editor, "Scientific Ballooning Handbook," NCAR TN/1A-99, National Center for Atmospheric Research, Boulder CO, 1975, Chaps. 3, 11,12 